\title{
Dynamic Failure of Composite Materials
}

\author{
J. LeBlanc ${ }^{1}$ A. Shukla ${ }^{2}$ Y. D. S. Rajapakse ${ }^{3}$
}

/ Published online: 21 August 2018

(c) This is a U.S. government work and its text is not subject to copyright protection in the United States; however, its text may be subject to foreign copyright protection 2018

The continually increasing demands of structural requirements and performance are driving a large number of industries to seek out the benefits of advanced material systems such as composite materials. These materials are finding increased roles in applications ranging from aerospace, marine, infrastructure, defense, and consumer applications. The benefits afforded by these novel material systems include high performance to weight ratios, overall reductions in maintenance, ability to manufacture complex geometries, and improved performance in aggressive environmental conditions. With the increased use of these materials, consideration must also be given to the extreme loading conditions which are imparted to them in the expected operating theatres. A fundamental understanding is needed of the material response when exposed to conditions such as, but not limited to, Impact, Shock, Hydrostatic Pressures, and Temperature extremes. It is with these considerations and goals at the forefront, that this special issue of the Journal of Dynamic Behavior of Materials is compiled with the specific focus on the Dynamic Failure of Composite Materials. The goal of this dedicated issue is to bring forth recent and quality research findings as they relate to the failure of these materials when subjected to a wide range of extreme conditions including: (1) Impact, (2) Blast, and (3) Low Temperatures. Additional studies on the fundamental performance of these materials under shear, axial, and biaxial loading are presented. Studies involving both experimental and computational methods are included in the contents of the issue.

The first section of this special issue presents studies on the characterization of materials under dynamic and low temperature conditions. The response of textile composites under dynamic tensile and bi-axial tension are presented by Justusson et al., while Cavallaro et al. examine the effects of low

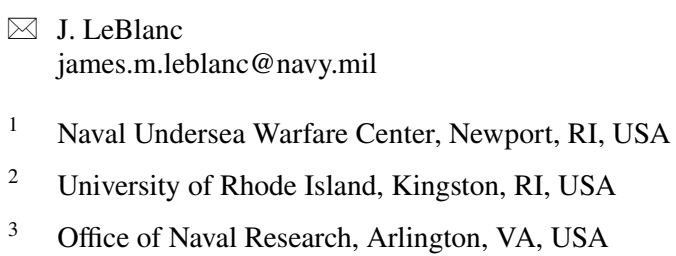

temperatures on the fracture properties of Kevlar laminates. Additionally, the work of Tessema and colleagues details the effects of nano-fillers on the shear properties of Carbon Fiber based materials. The second primary focus area of the issue is on the low velocity impact performance of composite materials, including studies which evaluate the effects of low temperatures. Research conducted by Castellanos et al. examines the effects of low temperature on the impact response of woven carbon laminates, while an investigation by Elamin et al. focuses on stitched and un-stiched composites under low temperature impact. Furthermore, Lopresto and colleagues examined the impact performance of Basalt Fiber based laminates to low velocity impact. Miao and Tippur discuss the implementation of an advanced Digital Gradient Sensing (DGS) method as a means for quantifying the slope of a plates surface while undergoing impact loading. Studies pertaining to High Speed Impact and Blast loading are presented in the third grouping of papers. Rolfe et al. present findings of face-sheet materials and construction on the high velocity impact and blast loading of sandwich panels. Similarly, Ballistic impact loading results, including numerical modeling, for S-2 Glass laminates are presented by Key and Alexander. Hufner et al. present the development of a novel test facility for the simulation of pressure loadings representative of underwater explosions, with corresponding computational results. The effects of air blast on the residual compressive strength of E-Glass laminates, including the development of a predictive formulation, are provided by Black et al. The final topic area in the issue evaluates the effects of a large hydrostatic pressure environment on composite cylinders. Shukla et al. present key findings on the physical phenomena of anisotropic cylinders which collapse under pressure in a limited energy environment.

In conclusion, this special issue is dedicated to presenting state of the art research on the dynamic failure of composite materials under a wide multitude of loading conditions. The editors wish to thank all authors for their significant contributions to the field of composite materials, and specifically to the success of this issue. Gratitude is also extended to the Journal of Dynamic Behavior of Materials as well as the Society for Experimental Mechanics for the opportunity to publish this work. 